\title{
ANÁLISE DE ATRIBUTOS FÍSICOS E QUÍMICOS DE SOLO SUBMETIDO A DIFERENTES MANEJOS NO SUDESTE PARAENSE
}

\author{
Fabrício Marinho Lisbôa ${ }^{1}$; Priscila Batista Miranda² \\ ${ }^{1}$ Doutorando do Programa de Pós-graduação em Produção Vegetal da Universidade Estadual do Norte \\ Fluminense Darcy Ribeiro. Campos do Goytacazes, Rio de Janeiro, Brasil. fabriciomarinho87@yahoo.com. \\ ${ }^{2}$ Eng. Agrônoma, Faculdade de Ciências Agrárias de Marabá, Universidade Federal do Sul e Sudeste do Pará. \\ Marabá, Pará, Brasil. engagro.priscila@ outlook.com.
}

RESUMO: Com intuito de avaliar propriedades físicas e químicas do solo, submetido a diferentes manejos, em propriedade agrícola familiar do sudeste paraense, coletou-se amostras de solo em áreas de mata primária (MP), pastagem com leguminosas (PL) e pastagem solteira (PS) em duas profundidades $(0$ a 5 e 5 a $10 \mathrm{~cm})$. Foram realizadas análises físicas: granulometria, Dp, Ds e Ptotal, assim como análises químicas: $\mathrm{pH}, \mathrm{Al}^{3+}, \mathrm{Ca}^{2+}, \mathrm{Mg}^{2+} \mathrm{e}^{+}$ trocáveis, $\mathrm{K}^{+}$e $\mathrm{P}, \mathrm{H}+\mathrm{Al}$ e COT. Para verificar a significância das diferenças entre as médias dos tratamentos foi aplicado o teste Tukey a 5\% de probabilidade, fazendo uso de delineamento inteiramente casualizado, com três tratamentos (MP, PL e PS) e três repetições, as profundidades foram estudadas separadamente. PS apresentou maiores valores para DS e menores valores para Ptotal na profundidade 0 a $5 \mathrm{~cm}$. Porém, na profundidade 5 a $10 \mathrm{~cm}, \mathrm{PL}$ e PS não apresentaram diferença para esses atributos. PL apresentou o maior valor para $\mathrm{pH}$ do solo e também menores valores para $\mathrm{Al}^{+3}$ e $\mathrm{H}+\mathrm{Al}$. Não houve diferença significativa entre os tratamentos, e profundidade, para $\mathrm{P}, \mathrm{K}^{+}, \mathrm{Ca}^{+2}$ e $\mathrm{Mg}^{+2}$, já o $\mathrm{Na}^{+}$diferiu apenas na primeira profundidade, com MP apresentando o maior valor. MP apresentou maiores teores de COT.

PALAVRAS-CHAVE: Agricultura familiar. Leguminosas. Pastagem. Solo.

\section{ANALYSIS OF PHYSICAL AND CHEMICAL PROPERTIES OF SOIL UNDER DIFFERENT MANAGEMENTS IN SOUTHEAST OF PARA STATE}

\begin{abstract}
In order to assess physical and chemical soil properties under different managements, on the family farm, in southeast of Para state, it was collected soil samples in areas of primary forest (PF), pasture with legumes (PL) and single pasture (SP) at two depths ( 0 to 5 and 5 to $10 \mathrm{~cm}$ ). Physical analyzes: particle size, Dp, Ds and Ptotal, as well as chemical analysis: $\mathrm{pH}$ in water, $\mathrm{Al}^{3+}, \mathrm{Ca}^{2+}, \mathrm{Mg}^{2+}$ and exchangeable $\mathrm{Na}^{+}, \mathrm{K}^{+}$and $\mathrm{P}, \mathrm{H}+\mathrm{Al}$ and TOC. To test the significance of differences between treatment means, we applied the Tukey test at 5\% probability, making use of completely randomized design with three treatment (PF, PL and SP) and three replications, the depths were studied separately. SP showed higher values for DS and Ptotal for depth 0 to $5 \mathrm{~cm}$. However, the depth 5 to $10 \mathrm{~cm}$, PL and SP did not differ for these attributes. PL had the highest value for soil $\mathrm{pH}$ and also lower values for $\mathrm{Al}^{3+}$ and $\mathrm{H}+\mathrm{Al}$. There was no significant difference between treatments, and depth, for $\mathrm{P}, \mathrm{K}^{+}$, $\mathrm{Ca}^{2+}, \mathrm{Mg}^{2+} . \mathrm{Na}^{+}$has differed only in the first depth, with $\mathrm{PF}$ having the highest value. PF presented the highest value to TOC.
\end{abstract}

KEYWORDS: Family farming. Legumes. Pasture. Soil. 


\section{ANÁLISIS DE LAS PROPIEDADES FÍSICAS Y QUÍMICAS DEL SUELO BAJO DIFERENTES MANEJOS EN EL SUDESTE DE PARÁ}

RESUMEN: Con el fin de evaluar las propiedades físicas y químicas del suelo bajo diferentes manejos, en la granja familiar sudeste de Pará, se recogió muestras de suelo en zonas de bosque primario (MP), pasturas con leguminosas (PL) y solo el pastoreo (PS) a dos profundidades (0 a 5 y de 5 a $10 \mathrm{~cm}$ ). Análisis físico: tamaño de partícula, Dp, Ds y Ptotal, así como análisis químicos : pH, $\mathrm{Al} 3+, \mathrm{Ca} 2+, \mathrm{Mg} 2+\mathrm{y} \mathrm{Na}$ intercambiable $+, \mathrm{K}+\mathrm{y} \mathrm{P}, \mathrm{H}+\mathrm{Al}$ y TOC. Para probar la significación de las diferencias entre las medias de los tratamientos se aplicó la prueba de Tukey al $5 \%$ de probabilidad, usando diseño completamente al azar con tres tratamientos (MP, PL y PS) y tres repeticiones, las profundidades se estudiaron por separado. PS mostró valores más altos para DS y los valores más bajos para Ptotal a $0-5 \mathrm{~cm}$ de profundidad. Sin embargo, la profundidad de 5-10 cm, PL y PS no difirieron para estos atributos. PL tuvo el valor más alto para el $\mathrm{pH}$ del suelo y valores también bajos de $\mathrm{Al} 3+\mathrm{y} \mathrm{H}$ + Al. No hubo diferencia significativa entre los tratamientos, y la profundidad, para $\mathrm{P}, \mathrm{K}+$, $\mathrm{Ca} 2+$ y $\mathrm{Mg} 2+, \mathrm{Na}+$ desde difiere sólo en la primera profundidad, con MP presentando el valor más alto. MP tuvo el mayor TOC.

PALABRAS-CLAVE: Agricultura familiar. Leguminosas. Pastoreo. Suelo.

\section{INTRODUÇÃO}

$\mathrm{Na}$ agricultura familiar amazônica é comum a alteração do meio ambiente natural para criação de áreas agrícolas, das quais se pode destacar a formação de pastagem proveniente da derrubada $\mathrm{e}$ queima da floresta. Após sua formação, o fogo é utilizado pelos agricultores como forma de controle de espécies espontâneas que surgem nessas pastagens como consequência de um processo que inclui a falta de manejo adequado tanto da forrageira principal quanto do solo.

Segundo Redin et al. (2011), a queima das pastagens pode ocasionar prejuízos físicos, químicos e biológicos ao solo. As principais alterações físicas podem ocorrer pela diminuição do volume de macroporos, do tamanho de agregados e da taxa de infiltração de água.

No que diz respeito aos atributos químicos, o aumento na disponibilidade de $\mathrm{P}$ e no teor de bases trocáveis são observados imediatamente após a queima, em consequência do acúmulo de cinzas provenientes da queima do material vegetal, os quais diminuem com o tempo devido a lixiviação dos nutrientes (KNICKER et al., 2006).

Em relação aos efeitos sobre os atributos biológicos do solo, Pomianoski et al. (2006) estudaram o efeito do fogo sobre essas características e concluíram em seu estudo que as queimadas influenciaram a quantidade de carbono da biomassa microbiana especialmente nas camadas de 1 a $2 \mathrm{~cm}$ do solo. 
Estudar as dinâmicas das propriedades dos solos é um processo importante em áreas em que o solo é submetido a diferentes práticas, pois pode indicar alternativas de manejo além de possibilitar estimativas de respostas dos atributos do solo em função de determinadas práticas realizadas (SANTOS, A. et al., 2009).

Nesse sentido, o objetivo desse trabalho foi analisar, física e quimicamente, propriedades de solo sob mata e pastagens submetidas a diferentes manejos em propriedade agrícola familiar do sudeste paraense.

\section{MATERIAL E MÉTODOS}

A coleta das amostras de solo ocorreu na propriedade "Três Irmãos", situada no $\begin{array}{lllll}\mathrm{km} & 90 \text { da rodovia } & \text { BR } 230\end{array}$ (Transamazônica), Itupiranga, Pará. O solo foi classificado como CAMBISSOLO HÁPLICO (EMBRAPA, 2009).

O clima regional é do tipo Am no limite de transição para Aw com temperatura média anual de $26,35^{\circ} \mathrm{C}$, caracterizado por um período menos chuvoso entre os meses de maio a outubro e um período mais chuvoso entre os meses de novembro a abril (KOPPEN, 1948).

As amostras foram coletadas em junho de 2013, em três áreas: Área de mata primária (MP); Área de pastagem com leguminosas (PL) e Área de pastagem solteira (PS), sendo três repetições e duas profundidades (0 a 5 e 5 a $10 \mathrm{~cm}$ ).

MP é um fragmento de Floresta Amazônica, não alterado.

PL foi área de mata primária até 1993, ano em que foi convertida em pastagem. Recebeu manejo de queimadas sucessivas até 2009, ano em que foi restringido o acesso de animais, ao mesmo tempo em que leguminosas (Cajanus cajan e Crotalaria juncea) foram inseridas. Em 2013 foi liberado ao acesso dos animais.

PS também foi área de mata primária até 1993, ano em que também foi convertida em pastagem. Recebeu manejo de queimadas sucessivas até 2013 e apresentou elevada presença de espécies espontâneas.

As duas pastagens contaram com uma lotação de 1,44 UA.ha-1 em pastejo contínuo, as mesmas nunca receberam calagem e/ou adubação.

Foram realizadas análises físicas do solo, como: análise granulométrica, pelo método da pipeta, agitação lenta (50 rpm) com agitador tipo Wagner e presença de dispersante químico $\left(\mathrm{NaOH} \mathrm{0,1} \mathrm{mol} \mathrm{L} \mathrm{L}^{-1}\right)$, com coleta da fração silte+argila (RUIZ, 2005).

Amostras indeformadas do solo foram coletadas em anéis volumétricos, os quais tiveram suas alturas e diâmetros mensurados. Foram levados à estufa a 105 ${ }^{\circ} \mathrm{C}$, pesados, esvaziados e pesados 
novamente (vazios) para determinação da Densidade do solo (Ds).

O solo seco, anteriormente nos anéis, foi passado em peneira de $2 \mathrm{~mm}$ de malha para determinação da densidade de partículas (Dp) pelo método do balão volumétrico (RUIZ, 2004).

Tabela 1 - Caracterização granulométrica e valores de densidade de partículas para os solos avaliados.

\begin{tabular}{|c|c|c|c|c|c|c|}
\hline Tratamento & $\mathrm{AG}$ & $\mathrm{AF}$ & Silte & Argila & \multicolumn{2}{|c|}{ Dp } \\
\hline & & ----- & g.kg ${ }^{-1}$ & - & & \\
\hline MP & & 0,422 & 0,161 & 0,118 & 0,289 & 2,70 \\
\hline PL & & 0,631 & 0,215 & 0,027 & 0,126 & 2,76 \\
\hline PS & & 0,329 & 0,224 & 0,125 & 0,324 & 2,87 \\
\hline
\end{tabular}

AG: areia grossa; AF: areia fina; Dp: densidade de partículas.

Foram realizadas análises químicas, como: $\mathrm{pH}$ em água, $\mathrm{Al}^{3+}, \mathrm{Ca}^{2+}, \mathrm{Mg}^{2+} \mathrm{e} \mathrm{Na}^{+}$ trocáveis, extraídos com $\mathrm{KCl} 1 \mathrm{~mol} \mathrm{~L}^{-1}$, na proporção $1: 10$, sendo $\mathrm{Al}^{3+}$ determinado por titulação com $\mathrm{NaOH} 0,025 \mathrm{~mol} \mathrm{~L}^{-1}$, $\mathrm{Ca}^{2+}$ e $\mathrm{Mg}^{2+}$ por espectrofotômetro de absorção atômica e $\mathrm{Na}^{+}$por fotometria de chama; $\mathrm{K}^{+}$e $\mathrm{P}$ disponíveis por extração com Mehlich-1, na proporção 1:10, dosados por fotometria de chama e colorimetria, respectivamente; $\mathrm{H}+\mathrm{Al}$ utilizando $\mathrm{Ca}(\mathrm{OAc})_{2}$ 0,5 $\mathrm{mol} \mathrm{L}^{-1}$, ajustado pH 7,0, na proporção 1:15, titulado com $\mathrm{NaOH}$ 0,025 $\mathrm{mol} \mathrm{L}^{-1}$ (DONAGEMMA et al., 2011).

A determinação do carbono orgânico total (COT) por oxidação via úmida (MENDONÇA; MATOS, 2005).
A porosidade total foi calculada pela expressão: $\mathrm{Pt}=1-\mathrm{Ds} / \mathrm{Dp}$, conforme Ruiz (2004). A caracterização granulométrica e valor de densidade de partículas encontram-se na Tabela 1. 
total. Nas áreas avaliadas PS apresentou maior valor de densidade do solo em 0 a 5 $\mathrm{cm} \quad(\mathrm{P}<0,05)$, com consequente menor valor para porosidade total (Tabela 2).

Tabela 2 - Densidade do solo e porosidade total de solo submetido a diferentes manejos, em três profundidades.

\begin{tabular}{|c|c|c|}
\hline Tratamento & Ds $\left(\mathrm{kg} \cdot \mathrm{dm}^{-3}\right)$ & Ptotal $\left(\mathrm{m} \cdot \mathrm{m}^{-3}\right)$ \\
\hline \multicolumn{3}{|c|}{0 a $5 \mathrm{~cm}$} \\
\hline MP & $1,68^{\mathrm{b}}$ & $0,358^{\mathrm{a}}$ \\
\hline PL & $1,71^{\mathrm{b}}$ & $0,375^{\mathrm{b}}$ \\
\hline PS & $1,89^{\mathrm{a}}$ & $0,346^{\mathrm{c}}$ \\
\hline \multicolumn{3}{|c|}{5 a $10 \mathrm{~cm}$} \\
\hline MP & $1,66^{\mathrm{b}}$ & $0,399^{\mathrm{a}}$ \\
\hline PL & $1,84^{\mathrm{a}}$ & $0,334^{\mathrm{b}}$ \\
\hline PS & $1,81^{\mathrm{a}}$ & $0,360^{\mathrm{b}}$ \\
\hline
\end{tabular}

Médias seguidas de mesma letra na coluna não diferente estatisticamente entre si $(\mathrm{P}<0,05)$ pelo teste de Tukey a $5 \%$ de probabilidade.

As camadas superficiais dos solos sob pastagens podem apresentar restrições físicas, como maior densidade e porosidade total em virtude do elevado tráfego animal, ocasionado pelo uso de taxas de lotação inadequadas para a capacidade de suporte das pastagens (SANTOS et al., 2010).

Na profundidade 5 a $10 \mathrm{~cm}$, o efeito das leguminosas em PL não foi significativo $(\mathrm{P}>0,05)$ o suficiente para distinguir as duas áreas de pastagens pelo teste de Tukey a 5\%. Porém o solo sob mata apresentou menores valores para os atributos físicos avaliados $\quad(\mathrm{P}<0,05)$ (Tabela 2) o que pode indicar melhores condições do solo sob mata, com maior diversidade biológica e maior teor de matéria orgânica, que são fatores responsáveis pela manutenção das condições físicas do solo, os quais também apresentam grande influência na reversão do estado de compactação do mesmo (SANTOS; SALCEDO, 2010).

Marques et al. (2013) após avaliar fisicamente um Latossolo Amarelo distrófico na região de Capitão Poço, Pará, também encontraram maiores valores para densidade do solo nas camadas superficiais, o mesmo encontrava-se em pousio, com cobertura de espécies espontâneas.

Com relação aos atributos químicos (Tabela 3), para $\mathrm{pH}$ em água, houve diferença significativa $(\mathrm{P}<0,05)$ entre os tratamentos nas diferentes profundidades. Na profundidade 0 a $5 \mathrm{~cm}$ o tratamento PL apresentou o maior valor para $\mathrm{pH}$, o que se repetiu em 5 a $10 \mathrm{~cm}$. 
Os valores de fósforo também não diferiram entrem os tratamentos $(\mathrm{P}>0,05)$ e, entre as profundidades (Tabela 3).

A não variação de fósforo disponível em ambientes sob diferentes coberturas vegetais, pode estar relacionada com sua ligação à óxidos de ferro e alumínio formando complexos de esfera interna, essas ligações refletem na estabilidade do fósforo em solos intemperizados de clima tropical (CARDOSO et al., 2011).
Não houve diferença para os íons $\mathrm{K}^{+}$, $\mathrm{Ca}^{+2}$ e $\mathrm{Mg}^{+2} \quad(\mathrm{P}>0,05)$, entre os tratamentos, em todas as profundidades. Em solos amazônicos, por estarem submetidos à elevados índices pluviométricos, o processo de lixiviação das bases trocáveis é intenso, agravando as perdas de nutrientes (COLLIER; ARAÚJO, 2010).

Tabela 3 - Fertilidade de Solo submetido a diferentes manejos, em três profundidades.

\begin{tabular}{|c|c|c|c|c|c|c|c|c|}
\hline Tratamento & $\mathrm{pH}$ & $\mathrm{P}$ & $\mathrm{K}$ & $\mathrm{Na}$ & $\mathrm{Ca}$ & $\mathrm{Mg}$ & $\mathrm{Al}$ & $\mathrm{H}+\mathrm{Al}$ \\
\hline & $\mathrm{H}_{2} \mathrm{O}$ & \multicolumn{3}{|c|}{-------'mg.dm"3----- } & \multicolumn{4}{|c|}{ 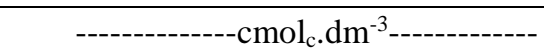 } \\
\hline \multicolumn{9}{|c|}{0 a $5 \mathrm{~cm}$} \\
\hline MP & $4,59^{b}$ & $0,55^{\mathrm{a}}$ & $81,33^{\mathrm{a}}$ & $46,33^{\mathrm{a}}$ & $3,42^{\mathrm{a}}$ & $2,96^{\mathrm{a}}$ & $0,76^{\mathrm{a}}$ & $4,90^{\mathrm{a}}$ \\
\hline PL & $5,65^{\mathrm{a}}$ & $0,47^{\mathrm{a}}$ & $38,33^{\mathrm{a}}$ & $40,00^{\mathrm{b}}$ & $2,84^{\mathrm{a}}$ & $2,54^{\mathrm{a}}$ & $0,31^{\mathrm{b}}$ & $2,25^{b}$ \\
\hline PS & $4,82^{\mathrm{b}}$ & $0,72^{\mathrm{a}}$ & $47,00^{\mathrm{a}}$ & $40,33^{\mathrm{b}}$ & $3,97^{\mathrm{a}}$ & $3,04^{\mathrm{a}}$ & $0,65^{\mathrm{a}}$ & $4,35^{\mathrm{a}}$ \\
\hline \multicolumn{9}{|c|}{$5 \mathrm{a} 10 \mathrm{~cm}$} \\
\hline MP & $4,25^{\mathrm{b}}$ & $0,50^{\mathrm{a}}$ & $71,66^{\mathrm{a}}$ & $45,00^{\mathrm{a}}$ & $1,32^{\mathrm{a}}$ & $1,61^{\mathrm{a}}$ & $1,28^{\mathrm{a}}$ & $4,45^{\mathrm{a}}$ \\
\hline PL & $5,32^{\mathrm{a}}$ & $0,30^{\mathrm{a}}$ & $32,66^{\mathrm{a}}$ & $38,33^{\mathrm{a}}$ & $2,07^{\mathrm{a}}$ & $0,98^{\mathrm{a}}$ & $0,40^{\mathrm{b}}$ & $2,19^{\mathrm{b}}$ \\
\hline PS & $4,44^{\mathrm{b}}$ & $0,37^{\mathrm{a}}$ & $40,33^{\mathrm{a}}$ & $40,00^{\mathrm{a}}$ & $1,53^{\mathrm{a}}$ & $1,08^{\mathrm{a}}$ & $1,36^{\mathrm{a}}$ & $4,89^{\mathrm{a}}$ \\
\hline
\end{tabular}

Médias seguidas de mesma letra na coluna não diferente estatisticamente entre si $(\mathrm{P}<0,05)$ pelo teste de Tukey a $5 \%$ de probabilidade.

$\mathrm{O} \mathrm{Na}^{+}$diferiu $(\mathrm{P}<0,05)$ apenas na primeira profundidade, em que MP apresentou maior valor enquanto PL e PS não diferiram entre si $(\mathrm{P}>0,05)$. Esse resultado pode ser atribuído a proteção que a copa das árvores fornecem ao solo evitando com que o mesmo seja menos lixiviado que os demais. Apesar de $\mathrm{K}^{+}$,

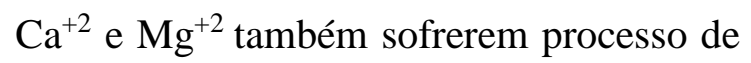
lixiviação, $\mathrm{K}^{+}$e $\mathrm{Na}^{+}$apresentam menor carga, sendo portanto, adsorvidos aos colóides minerais com menor força em relação ao demais. Porém, o $\mathrm{Na}^{+}$apresenta maior espessura do seu raio iônico hidratado do que $\mathrm{K}^{+}$, sendo adsorvido com menor força do que este (KINNIBURGH; JACKSON, 1981).

Em relação ao $\mathrm{Al}^{+3}$, MP e PS não diferiram entre si $(\mathrm{P}>0,05)$, apresentando os maiores valores em relação ao tratamento PL, o que também ocorreu para acidez potencial $(\mathrm{H}+\mathrm{Al})$ nas duas 
profundidades. Esse comportamento pode ser explicado pelo $\mathrm{pH}$, uma vez que, a atividade do alumínio na solução do solo pode ser controlada, entre outros fatores, pelo $\mathrm{pH}$ do meio, em solos com maiores valores de $\mathrm{pH}$ a atividade do $\mathrm{Al}^{+3}$ tende a ser menor (ZAMBROSI et al., 2007).
Os teores de carbono orgânico total (COT) foram maiores em MP, e menores em PS $(\mathrm{P}<0,05)$. Os teores em PL não diferiram entre as demais áreas $(\mathrm{P}>0,05)$ nas duas profundidades estudadas (Gráfico 1).

Gráfico 1 - Carbono orgânico total (COT) de solo submetido a diferentes manejos, em três profundidades.

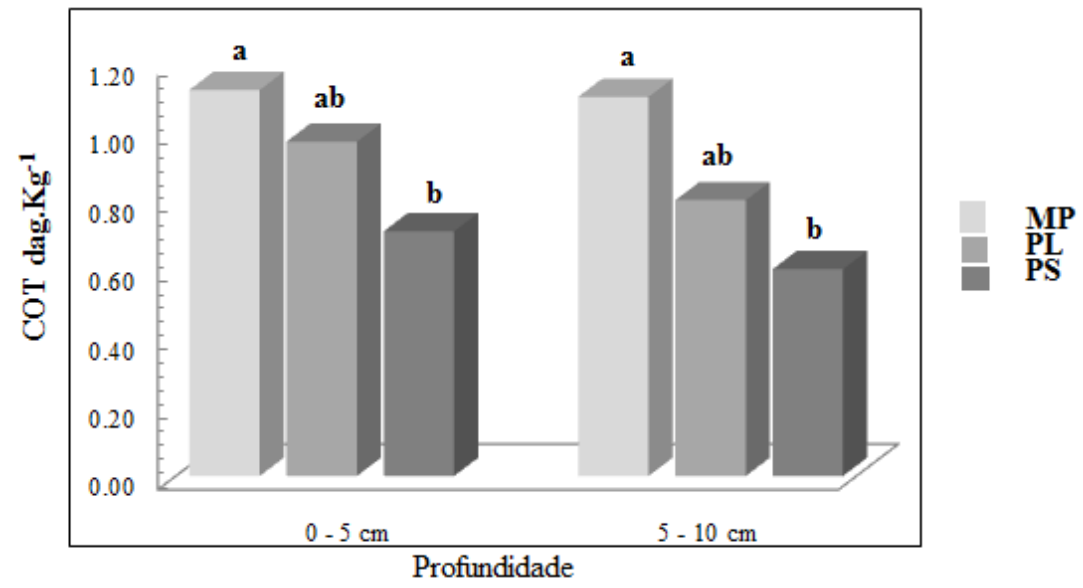

Médias seguidas de mesma letra não diferente estatisticamente entre si pelo teste de Tukey a $5 \%$ de probabilidade.

Em ambientes não alterados, os maiores teores de COT estão associados ao maior aporte, de forma contínua e diversificada, de material orgânico com diferentes graus de suscetibilidade à decomposição, provenientes de uma vegetação com maior diversidade de espécies como em MP (CARDOSO et al., 2011).

Como PL não diferiu das demais áreas, isso pode indicar efeito benéfico das leguminosas na restituição dos teores de COT a valores próximos de mata.

\section{CONCLUSÕES}

$\mathrm{Na}$ profundidade de 0 a $5 \mathrm{~cm}$, a pastagem solteira (PS) apresentou condições físicas inferiores às áreas com manejos mais conservacionistas (MP e PL), para os atributos densidade do solo e porosidade total. No entanto, na profundidade 5 a $10 \mathrm{~cm}$, as pastagens não 
apresentaram diferença entre si com MP apresentando as melhores condições em relação às demais.

$\mathrm{O}$ pH do solo teve influência na atividade do alumínio e da acidez potencial, com PL apresentando maiores valores para $\mathrm{pH}$ e menores para alumínio e acidez potencial.

De uma forma em geral, não houve diferença significativa para as bases $\left(\mathrm{K}^{+}\right.$, $\mathrm{Ca}^{+2}$ e $\mathrm{Mg}^{+2}$ ). Porém, MP apresentou maior valor para $\mathrm{Na}^{+}$em 0 a $5 \mathrm{~cm}$. $\mathrm{O}$ solo sob mata apresentou maiores valores de COT do que as pastagens.

\section{REFERÊNCIAS}

CARDOSO, E. L.; SILVA, M. L. N.; CURI, N.; FERREIRA, M. M.; FREITAS, D. A. F. Qualidade química e física do solo sob vegetação arbórea nativa e pastagens no pantanal sul-mato-grossense. Revista Brasileira de Ciência do Solo, v. 35, p. 613-622, 2011.

COLLIER, L. S.; ARAÚJO, G. P. Fertilidade de solo sob sistemas de produção de subsistência, agrofloresta e vegetação remanescente em EsperantinaTocantins. Revista Floresta e Ambiente, v. 17, p. 12-22, 2010.

DONAGEMMA, G. K.; CAMPOS, D. V. B.; CALDERANO, S. B.; TEIXEIRA, W. G.; VIANA, J. H. M. (Org.). Manual de métodos de análise de solo. Rio de Janeiro: CNPS, 2011. 225 p.

EMBRAPA. Sistema Brasileiro de Classificação de Solos. Rio de Janeiro: CNPS, 2009. 397 p.
FERREIRA, D. F. SISVAR: um programa para análises e ensino de estatística. Revista Symposium, v. 6, p. 36-41, 2008.

KINNIBURGH, D. G.; JACKSON, M. L. Cation adsorption by hydrous metal oxides and clay. In: ANDERSON, M. A. ; RUBIN, A. J. (Org.). Adsorption of Inorganics at Solid-Liquid Interfaces. Michigan: Ann Arbor Scientific, 1981, p. 91-160.

KNICKER, H.; ALMENDROS， G.; GONZÁLEZ-VILA, F. J.; POLVILLO, O. Characteristic alterations of quantity and quality of soil organic matter caused by forest fires in continental Mediterranean ecosystems: A solid-state ${ }^{13} \mathrm{C}$ NMR study. European Journal of Soil Science, v. 57, p. 558-569, 2006.

KÖPPEN, W. Climatología con un estudio de los climas de la tierra. México: FCE, 1948. 463 p.

MARQUES, D. C. S.; SILVA, D. M. S.; OLIVEIRA, S. S.; REIS, J. S.; COSTA, J. L. P.; SALDANHA, E. C. M. Atributos físicos avaliados em um Latossolo Amarelo em duas épocas do ano no município de Capitão Poço, PA. Revista Agroecossistemas, v. 5, p. 14-19, 2013.

MENDONÇA, E. S.; MATOS, E. S. Matéria Orgânica do Solo: Métodos de Análises. Viçosa: UFV, 2005. 81 p.

POMIANOSKI, D. J. W.; DEDECEK, R. A.; VILCAHUAMAN, L. J. M. Efeito do fogo nas características químicas e biológicas do solo no sistema agroflorestal da Bracatinga. Boletim de Pesquisa Florestal, v. 26, p. 93-118, 2006.

REDIN, M.; SANTOS, G. F.; MIGUEL, P.; DENEGA, G. L.; LUPATINI, M.; DONEDA, A.; SOUZA, E. L. Impactos da queima sobre atributos químicos, físicos e biológicos do solo. Ciência Florestal, v. 21, p. 381-392, 2011. 
RUIZ, H. A. Métodos de Análises Físicas

do Solo. Viçosa: UFV, 2004. 23 p.

Incremento da exatidão da análise granulométrica do solo por meio da coleta da suspensão (silte+argila). Revista Brasileira de Ciência do Solo, v. 29, p. 297-300, 2005.

SANTOS, A. C.; FERREIRA, E. M.; ARAÚJO, L. C. Propriedades físicas e químicas de solos em áreas sob pastagens em cerrado do norte do Tocantins. Revista Acadêmica: Ciências Agrárias e Ambientais, v. 7, p. 55-63, 2009.

SANTOS, A. C.; SALCEDO, I. H. Relevo e fertilidade do solo em diferentes estratos da cobertura vegetal na bacia hidrográfica da represa Vaca Brava, Areia, PB. Revista Árvore, v. 34, p. 277-285, 2010.

SANTOS, J. T.; ANDRADRE, A. P.; SILVA, I. F.; SILVA, D. S.; SANTOS, E. M.; SILVA, A. P. G. Atributos físicos e químicos do solo de áreas sob pastejo na micro região do Brejo Paraibano. Ciência Rural, v. 40, p. 2486-2492, 2010.

ZAMBROSI, F. C. B.; ALLEONI, L. R. F.; CAIRES, E. F. Teores de alumínio trocável e não trocável após calagem e gessagem em Latossolo sob sistema plantio direto. Bragantia, v. 66 , p. 487495, 2007. 